\title{
ESTUDO DAS FREQÜÊNCIAS DOS PRINCIPAIS FATORES DE RISCO PARA ACIDENTE VASCULAR CEREBRAL ISQUÊMICO EM IDOSOS
}

\author{
Sueli Luciano Pires', Rubens José Gagliardi², Milton Luiz Gorzoni
}

\begin{abstract}
RESUMO - Foram estudados retrospectivamente 262 pacientes com diagnóstico clínico de acidente vascular cerebral isquêmico (AVCi) permanente, com idade igual ou superior a 60 anos, selecionados dos 1015 registros da Liga de Aterosclerose da Clínica Neurológica da ISCMSP, de 1990 a 2002. O estudo focalizou as freqüências dos fatores de risco modificáveis para AVCi nesta população idosa, considerando-se sexo e faixa etária dos pacientes. Os resultados evidenciaram que a hipertenção arterial sistêmica é significativamente freqüente $(87,8 \%)$ entre pacientes idosos com AVCi, independentemente do sexo e da faixa etária. Tabagismo $(46,9 \%)$ e etilismo $(35,1 \%)$ revelaram-se fatores de riscos modificáveis freqüentes especialmente entre os homens. As cardiopatias (27,0\%), o Diabete Melito (19,9\%) e as dislipidemias (15,6\%) também se revelaram fatores de risco modificáveis freqüentes em pacientes idosos com AVCi, em ambos os sexos e em ambas as faixas etárias estudadas ( 60 a 70 anos e mais que 71 anos). Foi relativamente baixa a freqüência de hiperuricemia nesta amostra.
\end{abstract}

PALAVRAS-CHAVE: acidente vascular cerebral isquêmico, idosos, AVC, fatores de risco.

\begin{abstract}
Study of the main risk factors frequencies for ischemic cerebrovascular disease in elderly patients
ABSTRACT - Two hundred and sixty two patients with clinical diagnosis of permanent ischemic stroke, all of them aged 60 or more were retrospectively studied from the 1015 cerebrovascular diseases (CVD) records of the Atherosclerosis Ligue of the Neurology Clinics of the ISCMSP, from 1990 to 2002. The study emphasized modifiable risk factors frequencies for ischemic stroke in this population, considering gender and age of the patients. Results have evidenced that systemic arterial hypertension is a main risk factor significantly frequent in old people (87.8\%), independently of gender and age. Smoking (46.9\%) and alcohol consumption (35.1\%) have revealed to be very frequent important modifiable risk factors especially among men. Lower frequencies have been presented for cardiac diseases (27.0\%), Diabetes Melitus (19.9\%), and dislipidemia (15.6\%) as risk factors for ischemic stroke in old people of both genders and all ages after 60. There was relatively low frequency of hiperuricemia in this set of patients.
\end{abstract}

KEY WORDS: ischemic stroke, elderly patients, stroke, risk factors.

As doenças do aparelho circulatório acarretam taxas de mortalidade proporcional de $32,3 \%$, e constituem as principais causas de óbito no Brasil. Este grupo é liderado pela doença cerebrovascular (DCV), que é responsável por um terço das mortes. Em 1996, o índice de mortalidade por DCV foi 56,1 por 100000 habitantes ${ }^{1}$. A DCV representa $8,2 \%$ das internações e $19,0 \%$ dos custos hospitalares do Instituto Nacional de Assistência Médica da Previdência Social - INAMPS². Nos Estados Unidos da América, cerca de $50,0 \%$ das internações relativas a casos neurológicos decorrem de $\mathrm{DCV}^{3}$. Dentre as
DCV, destaca-se o acidente vascular cerebral (AVC), que pode se apresentar de formas variadas, ter diferentes manifestações clínicas e etiologias diversas ${ }^{4,5}$. O AVC isquêmico (AVCi), ou seja, o déficit neurológico resultante da insuficiência de suprimento sangüíneo cerebral, que pode ser transitório (episódio isquêmico transitório) ou permanente, representa, na população nacional, segundo diferentes estatísticas, de $53,0 \%^{6}$ a $85,0 \%^{6-8}$ dos casos de AVC, predominando a sua forma permanente. A detecção e o controle dos fatores de risco são tarefas prioritárias, pois permitem redução significativa da inci-

\footnotetext{
'Médica Geriatra Assistente da Disciplina de Gerontologia, da Faculdade de Ciências Médicas da Santa Casa de São Paulo Brasil (FCMSCSP), Diretora Técnica do Hospital Geriátrico e de Convalescentes D. Pedro II, Mestre em Medicina pela FCMSCSP; ${ }^{2}$ Professor Adjunto do Departamento de Clínica Médica da Disciplina de Neurologia, da FCMSCSP, Doutor em Neurologia pela Universidade de São Paulo; ${ }^{3}$ Professor Adjunto do Departamento de Clínica Médica, da Disciplina de Gerontologia, da FCMSCSP, Doutor em Medicina pela FCMSCSP.
}

Recebido 9 Abril 2003, recebido na forma final 30 Março 2004. Aceito 20 Maio 2004.

Dra. Sueli Luciano Pires - Rua Azevedo Soares 425/51 - 03322-000 São Paulo SP - Brasil. E-mail: suelipires@directnet.com.br 
dência e recidiva do AVCi, cuja taxa de mortalidade varia de $14,0 \%$ a $26,0 \%$, por intermédio de mudanças de hábitos de vida, terapêutica medicamentosa, neuro-radiologia intervencionista ou cirurgia. A sua etiologia é outro ponto que deve ser avaliado, pois possibilita tratamento e/ou correção adequados, reduzindo o risco de recidiva ${ }^{3,8-17}$. Ressalte-se, ainda, que grande parte da população não tem esta condição devidamente diagnosticada; alguns fatores de risco ainda são desconhecidos, e em muitos doentes o AVCi não tem etiologia esclarecida5,6.

A hipertensão arterial sistêmica (HAS) é o principal fator de risco preditivo para AVCi, pois está presente em cerca de $70,0 \%$ dos casos de DCV ${ }^{6,7}$. Cardiopatias são consideradas o segundo fator de risco mais importante para AVC, cuja freqüência é 41,9\% para AVCi (contra cerca de 2,0\% para AVC hemorrágico). Fibrilação atrial crônica (FA) é a doença cardíaca mais associada com AVC, representando cerca de $22,0 \%$ destes casos $^{6}$. Diabete melito (DM) é fator de risco independente para a DCV, uma vez que acelera o processo aterosclerótico ${ }^{14}$. Cerca de $23 \%$ de pacientes com AVCi são diabéticos ${ }^{6}$.

As DCV incidem com maior freqüência na idade avançada, período de vida em que se observam as maiores taxas de óbito e seqüelas. $O$ doente idoso, comparado ao doente jovem, possui algumas características próprias em relação à etiologia e prevenção destas doenças. Há nítida predominância da aterosclerose como causa de DCV, ao contrário dos jovens, entre os quais prevalecem condições hereditárias, malformações e uso de drogas ilícitas. Há vários estudos sobre as causas e os fatores de risco de AVC em jovens; paradoxalmente, há menos

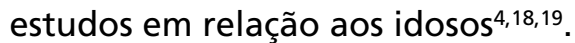

Com a finalidade de aprofundar estudos e pesquisas sobre DCV e aperfeiçoar o atendimento ao doente, a Clínica Neurológica do Hospital Central da Irmandade da Santa Casa de Misericórdia de São Paulo iniciou em 1990 os trabalhos da Liga de Aterosclerose que soma, hoje, 1015 casos de DCV registrados, dos quais foram separados os protocolos dos doentes idosos (idade igual ou maior que 60 anos) com AVCi em sua forma permanente para este estudo, cuja premissa geral consiste na análise das freqüências dos principais fatores de risco modificáveis para AVCi na população geriátrica, considerando também o sexo e a faixa etária dos pacientes.

\section{MÉTODO}

Foram incluídos neste estudo apenas os 262 pacientes que chegaram à Liga de Aterosclerose da Clínica Neurológica da Irmandade da Santa Casa de Misericórdia de São Paulo para cadastro e acompanhamento já com diagnóstico fechado de AVCi permanente e idade igual ou superior a 60 anos por ocasião do evento único ou do primeiro evento, selecionados dentre 1015 prontuários de doentes matriculados na mesma Liga de 1990 a 2002. Para esta seleção, foram primeiramente separadas as fichas de todos os pacientes com diagnóstico de $\mathrm{AVCi}$, especificamente em sua forma permanente. Destas fichas, foram então separadas para o estudo apenas as dos pacientes com 60 anos ou mais.

Para a observação das freqüências de fatores de risco modificáveis, o protocolo utilizado enfatizou: (1) dados de identificação do paciente, particularmente o sexo e a idade; (2) presença de fatores de risco para AVCi, destacando-se HAS (valores iguais ou maiores que $140 \mathrm{mmHg}$ por $90 \mathrm{mmHg}$ ), DM (três glicemias em jejum com valores acima de $126 \mathrm{mg} / \mathrm{dL}$ ), cardiopatias, antecedentes pessoais (tabagismo e etilismo); e (3) os exames laboratoriais, notadamente colesterol (total e frações), triglicérides e glicemia (jejum), segundo os métodos padronizados do Laboratório Central do Hospital Central da Irmandade da Santa Casa de Misericórdia de São Paulo. Para complementação de nossas observações e melhor caracterização deste grupo específico de pacientes, embora não tenham sido objetivos deste estudo, também interessaram ao protocolo de estudo (1) a história familiar de AVC; (2) os exames de imagem, principalmente tomografia computadorizada (TC) de crânio e ultra-sonografia (USG) das artérias carótidas, quando disponíveis; e (5) o eletrocardiograma.

Os dados foram inicialmente analisados descritivamente. O teste do Qui-quadrado $\left(\chi^{2}\right)$ foi utilizado para a comparação dos achados expressos em freqüências, de acordo com o sexo e a faixa etária (de 60 a 70 anos e mais de 71 anos). Dados expressos em médias foram analisados comparativamente com o teste $\mathrm{t}$ de Student. Adotou-se $p \leq 0,05$ (probabilidade de $95,0 \%$ ) para a interpretação dos resultados.

\section{RESULTADOS}

Dentre 1015 casos registrados de DVC, 262 $(25,8 \%)$ referiam-se a AVCi permanente em doentes com 60 anos ou mais, características essas que nortearam a seleção dos casos aqui apresentados. $\mathrm{Pa}$ cientes do sexo masculino representaram $52,7 \%$ desta amostra, com idades entre 60 e 93 anos (média etária de $67,7 \pm 6,7$ anos). Pacientes do sexo feminino, por sua vez, somaram $47,3 \%$, com idades entre 60 e 95 anos (média de idade de $68,5 \pm 7,0$ anos). A maioria dos pacientes de ambos os sexos apresentou faixa etária entre 60-70 anos (66,0\%).

A distribuição dos pacientes de acordo com o sexo e a faixa apresentou freqüências estatisticamente similares $\left(\chi^{2}=3,71 ; p>0,05\right)$. Observou-se, porém, que houve maior freqüência de mulheres com mais de 71 anos, contra maior freqüência de homens com 60-70 anos. As mulheres apresentaram 
média de idade $(68,5 \pm 7,0$ anos) ligeiramente superior do que os homens $(67,7 \pm 6,7)$, diferença essa que não se mostrou significativa à estatística ( $t=$ 0,$0635 ; p>0,05$ ).

A HAS foi encontrada em $87,8 \%$ dos doentes ( $85,5 \%$ dos homens e $90,3 \%$ das mulheres), não havendo diferença estatisticamente significativa nesta variável quanto ao sexo nem entre as faixas etárias. A maioria absoluta dos pacientes com HAS apresentou alteração dos valores tanto da pressão sistólica quanto da diastólica (181 casos, 78,0\%). Em oito casos (3,0\%), a hipertensão referia-se apenas à pressão arterial sistólica. Nos demais 41 casos $(15,6 \%)$, embora houvesse registro da presença de HAS nos protocolos, não estava determinado o tipo de pressão arterial alterada.

Constatou-se DM em 51 idosos (19,9\%), com freqüências similares para homens $(20,8 \%)$ e mulheres $(18,8 \%)$, e para os pacientes das diferentes faixas etárias estudadas. Em seis casos não havia registro sobre a presença ou não de DM.

A freqüência de pacientes que apresentavam cardiopatias $(27,0 \%)$ foi similar para homens $(28,3 \%)$ e mulheres $(25,8 \%)$. Nos pacientes com mais de 71 anos, a freqüência de cardiopatias $(31,5 \%)$ foi maior do que naqueles com idade entre 60 e 70 anos $(24,8 \%)$; todavia, essa diferença não se mostrou estatisticamente significativa $\left(\chi^{2}=1,99 ; p>0,05\right)$. Destes 71 pacientes, $58(81,7 \%)$ apresentavam cardiopatias isoladas, e os demais $13(18,3 \%)$ referiram duas ou mais condições cardíacas patológicas. Dentre as cardiopatias registradas, houve 23 casos $(32,4 \%)$ de infarto agudo do miocárdio prévio; 23 casos de arritmias (32,4\%), 22 dos quais se referiram a fibrilação atrial, e um caso a bloqueio atrioventricular total; 14 casos $(19,8 \%)$ de insuficiência cardíaca congestiva; e 14 casos $(19,8 \%)$ de sobrecarga ventricular esquerda (SVE). Houve 11 registros $(15,5 \%)$ de outras cardiopatias, que incluíram miocardiopatia (quatro casos), cardiopatia inespecífica (três casos), disfunção diastólica de ventrículo esquerdo (dois casos) e doença de Chagas (dois casos).

Foi registrada freqüência de $15,6 \%$ de pacientes com algum tipo de dislipidemia. Não houve diferença significativa entre os sexos nem entre faixas etárias com relação a esta variável.

Houve freqüência de $8,8 \%$ de pacientes com hiperuricemia. Não houve diferença significativa entre os sexos com relação a esta variável. Pacientes com mais de 71 anos apresentaram freqüência significativamente maior $\left(\chi^{2}=5,14 ; p<0,05\right)$ de hiperuricemia $(14,6 \%)$ do que aqueles com idade entre 60 e 70 anos $(5,8 \%)$.
Antecedentes de tabagismo foram registrados em $46,9 \%$ dos pacientes, freqüência essa significativamente maior $\left(\chi^{2}=21,34 ; p<0,05\right)$ entre os homens $(60,4 \%)$ do que entre as mulheres $(30,9 \%)$; mas não houve diferença significativa quando comparadas as faixas etárias. Dentre os casos de tabagismo, 58 pacientes $(50,0 \%)$ já haviam abandonado o hábito e $36(31,0 \%)$ ainda fumavam por ocasião do episódio de AVCi. Nos demais 22 protocolos $(19,0 \%)$ estava apenas registrada história de tabagismo, sem referência a ser pregressa ou corrente ao evento. Em 15 casos, não havia registro sobre a presença ou não de história de tabagismo.

A freqüência de etilismo foi de $35,1 \%$. Esta freqüência foi significativamente maior $\left(\chi^{2}=69,15\right.$; $p<0,05)$ entre os homens $(57,5 \%)$ do que entre as mulheres $(9,8 \%)$, assim como entre pacientes com menos de 70 anos $(40,6 \%)$ do que entre aqueles com mais de 71 anos $(24,4 \%)\left(\chi^{2}=4,06 ; p<0,05\right)$. Dos 84 pacientes com história de etilismo, 41 $(48,9 \%)$ referiram ter deixado de consumir álcool antes da ocorrência do AVCi; outros 22 (26,2\%) ainda consumiam álcool por esta ocasião. Nos demais 21 protocolos $(24,9 \%)$ só havia registro de história de etilismo, sem referência à atualidade ou não deste hábito. Em 23 casos, não havia registro sobre a presença ou não de história de etilismo.

História familiar de AVCi foi observada em 17,3\% dos casos. Não foram evidenciadas quaisquer diferenças estatisticamente significativas na distribuição das freqüências desta variável quando comparados os sexos nem quando comparadas as faixas etárias.

Com relação à distribuição das freqüências de acordo com os resultados de exames laboratoriais, as mulheres mostraram resultados alterados em colesterol total $(40,4 \%)$ e glicemia $(43,2 \%)$ com freqüência significativamente maior (respectivamente, $\chi^{2}=9,70 ; p<0,05$ e $\left.\chi^{2}=7,75 ; p<0,05\right)$ do que os homens ( $20,0 \%$ e $23,8 \%$, respectivamente). Os níveis de HDL mostraram-se alterados em número significativamente maior $\left(\chi^{2}=15,17 ; p<0,05\right)$ de homens $(39,2 \%)$ do que de mulheres $(14,5 \%)$. Não houve diferenças entre os sexos com relação às concentrações séricas de LDL, VLDL e triglicérides. Estas freqüências mostraram-se similares na comparação entre pacientes com menos de 70 anos e com mais de 71 anos, exceção feita pelos níveis de VLDL, que não estavam alterados em nenhum dos pacientes com mais de 71 anos.

O registro dos resultados dos exames laboratoriais mostrou que, para a amostra geral, as concentrações médias de colesterol total e frações (exceto LDL) e triglicérides eram normais, enquanto os de gli- 
Tabela 1. Valores mínimo, máximo, média \pm desvio padrão e mediana relativos aos registros de exames laboratoriais de 266 pacientes com AVCi (Liga de Aterosclerose da Clínica de Neurologia da ISCMSP, 1990-2002).

\begin{tabular}{lcccc}
\hline Parâmetros laboratoriais & Valor mínimo & Valor máximo & Média \pm DP & Mediana \\
\hline Colesterol total & 129 & 404 & $217,7 \pm 42,23$ & 210 \\
HDL & 13 & 87 & $42,2 \pm 12,6$ & 40 \\
LDL & 29 & 315 & $141,8 \pm 42,3$ & 139 \\
VLDL & 9 & 102 & $30,6 \pm 17,6$ & 27 \\
Triglicérides & 43 & 666 & $162,0 \pm 89,0$ & 142 \\
Glicemia & 71 & 377 & $114,9 \pm 45,8$ & 101 \\
\hline
\end{tabular}

Valores de Referência do Laboratório Central do Hospital Central da ISCMSP.

cemia e LDL-colesterol evidenciaram-se aumentados (Tabela 1). Não houve diferença entre os sexos quando comparados os níveis médios destes parâmetros laboratoriais, exceto pela LDL, cujo valor médio foi significativamente maior $(p=0,0021)$ entre as mulheres $(151,7 \pm 47,4 \mathrm{mg} / \mathrm{dL})$ do que entre os homens $(132,4 \pm 47,4 \mathrm{mg} / \mathrm{dL})$. Tampouco houve qualquer diferença digna de nota à estatística quando comparados os níveis laboratoriais médios dos pacientes com menos de 70 anos e aqueles com mais de 71 anos.

Em 137 (52,3\%) prontuários, não havia referência a achados de tomografia computadorizada, devido à simples falta de anotação, ao fato de o exame não ter sido realizado, ou porque o paciente, ainda que o tenha feito, não relatou respectivos resultados por ocasião de sua matrícula no serviço. Nos demais 125 prontuários (47,7\%) encontraramse resultados normais da TC em 18 casos $(6,9 \%)$ e anormais em 107 (40,8\%).

Em 107 prontuários (40,8\%) não havia registro de dados relativos à realização de ultra-sonografia de artérias carótidas, pelos mesmos motivos mencionados para a TC. Em 29 casos (11,0\%), os resultados deste exame de imagem mostraram-se normais, e nos demais 126 casos $(48,2 \%)$, anormais.

Não foram encontrados dados sobre a realização de eletrocardiograma em 208 prontuários (79,4\%), novamente pelas mesmas razões antes mencionadas. Em cinco casos (1,9\%) os resultados do eletrocardiograma eram normais; dois casos $(0,8 \%)$ referiram-se a doentes em uso de marca-passo; e nos demais 47 casos (17,9\%), os resultados mostraram-se anormais.

Dos 126 prontuários dos quais constavam alterações do sistema carotídeo à ultra-sonografia, em dez casos (7,9\%) não havia referência ao exato local da placa. Estas alterações eram unilaterais em 45 doentes $(35,8 \%)$ e bilaterais em $39(30,8 \%)$. Alterações uni ou bilaterais localizadas em diferentes sítios do sistema carotídeo estavam registradas em 32 casos $(25,5 \%)$. Com relação às localizações específicas das placas, foi evidenciado predomínio de alterações bilaterais nas artérias carótidas internas (17 casos) e bulbos (16 casos), imediatamente seguidas de alterações unilaterais das artérias carótidas internas direita (15 casos) ou esquerda (12 casos).

Não havia relato do tipo de placa observada ao ultra-som em 56 casos (44,4\%). Nos demais casos, tratava-se de placa calcificada em 31 casos $(24,6 \%)$; densa, em 21 (16,7\%); e mole, em 15 (11,9\%). Em três casos $(2,4 \%)$ havia referência a placas calcificadas e densas. Por fim, com relação ao grau de estenose da placa evidenciada à ultra-sonografia, esta informação não estava registrada em 44 prontuários (34,9\%). Em 36 casos (28,6\%) a placa causava estenose igual ou inferior a $30,0 \%$. Havia registro de estenoses iguais ou inferiores a 50,0\% em 19 casos (15,1\%). Em 16 casos (12,7\%), havia referência a espessamento intimal.

\section{DISCUSSÃO}

A Liga de Aterosclerose da Clínica de Neurologia do Hospital Central da Irmandade da Santa Casa de Misericórdia de São Paulo foi criada em 1990, com o intuito de desenvolver estudos e pesquisas sobre as DCVs e de aperfeiçoar o atendimento ao doente. Uma das tarefas de relevância da Liga tem sido o preenchimento de protocolo preestabelecido no qual, entre outras informações de interesse aos seus objetivos, constam dados obrigatórios para a investigação dos principais fatores de risco para as DCVs. Ressalte-se que os doentes cujos protocolos são cadastrados na Liga chegam já com as informações diagnósticas, clínicas ou laboratoriais; portanto, ainda não é função da Liga realizar qualquer avaliação diagnóstica, da DCV ou das doenças associadas, relacionadas como fatores de risco. A realização deste estudo, entretanto, revelou a necessidade de treinamento mais efetivo aos residentes 
que preenchem os cadastros, no sentido de os objetivos da Liga serem atingidos mais plenamente, orientando, inclusive, estudos retrospectivos que possam ser precisos, evitando-se lacunas nas informações que devem obrigatoriamente ser coletadas. Considerando que o AVC é a segunda causa de morte no mundo ocidental, que dois terços dos casos de $A V C$ se referem ao $A V C i$, e que a incidência deste quadro aumenta com o avanço da idade $\mathrm{e}^{4,7,13,20}$, foram selecionados, para este estudo, os 262 casos de AVCi em pacientes com 60 anos ou mais, selecionados dentre os 1015 casos de DCV registrados na referida Liga de Aterosclerose da Irmandade da Santa Casa de Misericórdia de São Paulo.

O interesse específico desta investigação foi o estudo das freqüências dos fatores de risco modificáveis para AVCi em idosos (HAS, DM, cardiopatias, dislipidemia, hiperuricemia, tabagismo e etilismo) bem documentados na literatura universal, conforme a classificação de Sacco et al..$^{20}$ relativamente à modificabilidade de fatores de risco para AVC. As demais informações contidas neste estudo serviram para completar o entendimento deste grupo específico de pacientes. Novos fatores de risco como homocisteína, paraoxonase, polimorfismos, fator $V$ de Leiden, proteína $C$, proteína $S$ e anticorpos antifosfolípides não foram analisados nesta casuística, uma vez que a coletânea dos dados abrangeu protocolos desde 1990, época em que alguns deles ainda não eram avaliados. Estes novos fatores de risco se referem principalmente ao AVC em jovens, o que não é o propósito da presente investigação. Além disso, não foi ainda a nossa intenção estudar o impacto desses fatores de risco, o que faremos oportunamente, em investigações adequadamente controladas.

Encontramos que $25,8 \%$ das DCVs registradas na Liga referem-se a AVCi permanente em pacientes com mais de 60 anos de idade. A literatura refere percentual de $53,0 \%{ }^{6}$ a $85,0 \%{ }^{7}$ de AVCi entre os AVCs na população geral, porém sem referência à idade dos pacientes. Se tivéssemos computado apenas os casos de pacientes com idade maior que 60 anos e retirado apenas deste grupo os casos de AVCi, certamente este percentual seria maior, aproximando-se, talvez, das freqüências indicadas na literatura. Esta discrepância entre percentuais talvez decorra também do fato de o diagnóstico dos casos de AVCi cadastrados na Liga ter sido realizado predominantemente com base em critérios clínicos, e percentual expressivo de pacientes não tem acesso imediato ou mediato a exames de imagem para a confirmação diagnóstica. Escalas e pontuações baseadas apenas em dados clínicos parecem ser ineficazes para distinguir AVC hemorrágico do AVC isquêmico, mesmo quando se consideram os fatores de risco e evento prévio de DCV na história clínica dos pacientes sob diagnóstico ${ }^{21}$. Estudos mais recentes e futuros, no entanto, já podem se beneficiar de nova escala de avaliação clínica para o diagnóstico diferencial entre AVCi e AVC hemorrágico proposta por Massaro 22 , possibilitando melhor padronização das casuísticas avaliadas.

$\mathrm{Na}$ literatura é referida maior incidência de AVC em homens ${ }^{4}$, o que não foi observado nesta série de pacientes. Tampouco foram encontradas diferenças entre os sexos quando se compararam pacientes com 60 a 70 anos e aqueles com mais de 71 anos, o que sugere que o maior risco de AVCi para homens deve aumentar com o avanço da idade até os 60 anos; a partir daí, o sexo dos doentes não parece interferir na freqüência dos casos de AVCi. Dados deste estudo evidenciaram que as mulheres tenderiam a apresentar AVCi em idade mais avançada do que os homens.

A HAS constitui o principal fator de risco modificável para $\mathrm{AVCi}$, com risco relativo de seis vezes de pacientes hipertensos desenvolverem $\mathrm{AVCi}^{23}$. Entre $67,5 \%{ }^{6}$ e $80,0 \%{ }^{7}$ dos pacientes com AVC são hipertensos, e entre $60,0 \%{ }^{6}$ e $70,0 \%{ }^{24}$ dos casos de AVCi estão associados com HAS. Nesta casuística, 87,8\% dos pacientes AVCi permanente eram hipertensos, e esta alta freqüência se justifica, uma vez que um dos principais fatores de risco para HAS é idade superior a 60 anos $^{8}$, muito embora já tenha se encontrado alta freqüência de HAS entre pacientes com AVCi com idades entre 15 e 49 anos $^{25}$. Na maioria dos nossos casos, tanto a pressão arterial sistólica quanto a diastólica estavam aumentadas. Apenas 3,0\% dos pacientes apresentavam apenas a pressão arterial sistólica aumentada, achado diferente do estudo clássico SHEP ${ }^{26}$, que salienta que a hipertensão sistólica é mais freqüente no idoso.

Embora a literatura indique maior prevalência de HAS entre homens ou entre mulheres após a menopausa ${ }^{27}$, não foram encontradas diferenças significativas nas freqüências de HAS quando comparamos os pacientes com relação ao sexo e às faixas etárias, o que parece confirmar a importância de medidas preventivas para HAS em toda a população, seja por meio de mudança de hábitos ou pela terapêutica medicamentosa adequada. Diversos estudos clínicos atestaram a eficácia do tratamento da HAS na redução do risco para AVCi em até $42,0 \%{ }^{26,28}$. 
Ressalte-se que o tabagismo, a dislipidemia (especialmente a hipercolesterolemia) e o DM constituem os fatores de risco mais críticos para a HAS, e o controle destes fatores deve ser igualmente cuidadoso. Note-se, ainda, que há medicamentos para o tratamento da HAS que podem piorar os demais fatores de risco, induzindo, por exemplo, hiperglicemia, dislipidemia ou alterações nos níveis de ácido úrico. Portanto, deve-se redobrar o cuidado com os pacientes com HAS que apresentem outros fatores de risco, pois pouco efeito surtirá o tratamento da HAS se as demais condições não forem consideradas ${ }^{26}$.

O risco relativo de pacientes com DM desenvolverem $\mathrm{AVCi}$ é de quatro vezes ${ }^{23}$, o que o coloca entre os principais fatores de risco para AVC. Tratase de condição que atua diretamente na parede endotelial, favorecendo a aterosclerose, ou indiretamente, já que é potente fator de risco para HAS $^{29}$. Nesta série, $19,9 \%$ dos casos de AVCi estavam associados com DM, sem quaisquer diferenças significativas com relação ao sexo nem com a faixa etária. Este percentual é compatível ao relatado pela literatura, que se aproxima de $15,0 \%{ }^{29}$.

Em exames laboratoriais a que tivemos acesso por ocasião do registro do paciente na Liga, as concentrações de glicemia em jejum apresentaram-se aumentadas em $32,4 \%$ dos pacientes. Esta freqüência foi maior entre as mulheres $(43,2 \%)$ do que entre os homens (23,8\%). Uma vez que $19,9 \%$ dos pacientes tinham diagnóstico confirmado de DM, e 32,4\% apresentaram elevação dos níveis de glicemia em jejum, parece lícito especular se o processo subjacente ao DM já não estaria interferindo na alta taxa de pacientes com HAS encontrada nesta amostra.

A freqüência de pacientes cardiopatas nesta casuística foi de $27,0 \%$, e estes pacientes geralmente apresentaram diferentes cardiopatias associadas. Infarto agudo do miocardio prévio constituiu a cardiopatia mais freqüente, somando $32,4 \%$ dos pacientes cardiopatas, ou 8,8\% da amostra geral. A segunda cardiopatia mais freqüente foram as arritmias, representadas, na quase totalidade dos casos, por FA $(8,4 \%)$. Este percentual está aquém da freqüência relatada na literatura, que chega a $36,0 \%$ dos casos de AVC, especialmente entre pacientes com 80 a 89 anos $^{30}$. O risco relativo de doentes com FA desenvolverem AVCi é de três ve$z^{2} s^{23}$. É possível que este achado não traduza a realidade da casuística populacional, pois, seguramente, muitos pacientes com FA são acompanhados no ambulatório de cardiologia, o que influencia na estatística deste estudo. Não foi observada qualquer diferença significativa à estatística nas freqüências de cardiopatias com relação ao sexo nem com relação às faixas etárias. No entanto, ficou evidente a tendência de tais condições serem mais freqüentes nos pacientes com mais de 71 anos. A doença de Chagas que, classicamente, é freqüente em nosso meio, foi rara nesta casuística, mostrando que, apesar de ser importante risco para os grupos de jovens, tem pequeno peso quando se analisam separadamente os pacientes idosos.

A dislipidemia é um dos principais fatores de risco para doenças vasculares, principalmente entre os pacientes diabéticos que, por sua vez, apresentam alta incidência de $\mathrm{HAS}^{28}$. Nesta amostra, 15,6\% dos casos de AVCi apresentavam a condição, sem diferenças quando analisados o sexo e a faixa etária dos pacientes. Ainda não está totalmente definida a relação entre AVC e dislipidemia que, com certeza, tem peso menos significativo comparativamente às doenças cardiovasculares ${ }^{20}$. Em estudo comparativo das frações de HDL e LDL colesterol como fatores de risco para a aterosclerose carotídea em 125 pacientes de ambos os sexos, com média de idade de 60 anos (entre 45 e 75 anos), concluiuse que níveis baixos de HDL seriam mais aterogênicos para as artérias carótidas do que níveis elevados de LDL ${ }^{31}$.

Paradoxalmente, ambas as teorias que explicam a fisiopatologia da aterosclerose referem-se a níveis aumentados de LDL. A primeira hipótese, a lípidica, defende que a formação do ateroma seria provocada pela elevação dos níveis séricos de LDL do colesterol, que penetraria no endotélio até a camada média, onde seria fagocitado por macrófagos, formando células espumosas que, em conjunto, originariam as estrias gordurosas que, por sua vez, constituem os elementos críticos para a formação do ateroma ${ }^{32}$. Segundo a outra hipótese, o processo teria início por lesão endotelial, e, dentre os principais agentes causadores desta lesão, estariam níveis elevados de $\mathrm{LDL}^{33}$. Na casuística como um todo, sem diferenças entre homens e mulheres nem entre as faixas etárias, a freqüência de resultados alterados para triglicérides foi de $20,0 \%$. Este dado reforça a proposta de que esta investigação deve ser procedida em todos os pacientes com mais de 60 anos, independentemente do sexo.

Também foi encontrada taxa de $8,8 \%$ de pacientes com hiperuricemia entre os casos de AVCi em pacientes com 60 anos ou mais. Embora este 
percentual tenha sido semelhante para homens e mulheres, foi significativamente maior nos pacientes com mais de 71 anos $(14,6 \%)$ do que entre aqueles com menos de 70 anos (5,8\%). Este achado deixa em aberto a necessidade de mais estudos, especialmente no que se refere a pacientes mais idosos. Embora a hiperuricemia possa ser causada por deficiências enzimáticas, sabe-se que, na maioria dos casos, é decorrente de defeito do transporte renal que acarreta depuração reduzida de ácido úrico, e ocorre predominantemente nos homens (10\% da população masculina pode apresentar ácido úrico elevado) e raramente nas mulheres. Além disso, o DM é causa não rara de disfunção renal; cerca de $26 \%$ dos pacientes que ingressam em programas de diálise são diabéticos ${ }^{29}$. Quatro $(17,0 \%)$ dos 23 pacientes desta amostra que apresentavam concentrações séricas alteradas de ácido úrico eram de fato diabéticos. Os níveis elevados de ácido úrico encontrados nesses pacientes certamente estão associados à taxa de diabéticos da casuística, bem como e, talvez, sobretudo, a defeitos do transporte renal oriundos de outras condições que se manifestam com o avanço da idade, incluindo uso de medicamentos, especialmente os anti-hipertensivos. Com exceção de três, todos os demais pacientes hiperuricêmicos $(87,0 \%)$ eram hipertensos e tratavam a HAS. Todos os pacientes com níveis elevados de ácido úrico também apresentavam concentrações séricas elevadas de uréia e creatinina, ratificando o papel da disfunção renal nos casos de hiperuricemia.

O tabagismo aumenta o risco de AVCi em duas vezes, e também constitui importante fator de risco para $\mathrm{HAS}^{8,20,23}$. Na década de 80 , a freqüência de tabagistas entre pacientes com AVC chegava a $80,0 \%{ }^{29}$. Com as campanhas mundiais contra o fumo, estas taxas caíram ${ }^{8}$, mas ainda são altas. Nesta série, 46,9\% dos pacientes com AVCi relataram história de tabagismo, freqüência que foi significativamente maior entre os homens, independentemente da faixa etária estudada. Alguns autores sugerem que o abandono do tabaco, especialmente em forma de cigarros, reduz o risco de AVC em dois a cinco anos ${ }^{20,30}$. Entretanto, considerando o índice de pacientes com AVCi que abandonaram o hábito antes do primeiro evento $(50,0 \%)$, infere-se que os males causados pelo tabaco persistem por longo tempo, especialmente quando o hábito é abandonado tardiamente.

O etilismo, especialmente o crônico e pesado, que é outro fator de risco modificável para AVCi, aumenta consideravelmente as taxas de morbidade e mortalidade nesta população ${ }^{11,34}$. Observamos 35,1\% de casos de etilismo entre os pacientes, também mais freqüente entre os homens e igualmente mais freqüente entre os pacientes com menos de 70 anos. Este achado está de acordo com os nossos padrões culturais, segundo os quais os homens tendem a consumir mais álcool do que as mulheres e a reduzir este vício com a idade avançada.

Embora o protocolo reserve espaço para tal informação, não dispusemos de dados que informassem o grau de etilismo dos pacientes deste estudo, razão pela qual foram considerados etilistas todos aqueles que relataram ingerir ou ter ingerido bebida alcoólica em quantidade e com freqüência sistemáticas. A contrapartida era os declarados abstêmios, ou seja, aqueles que não ingerem ou nunca ingeriram qualquer bebida alcoólica, exceto em raras ocasiões sociais. Curioso notar que, para o estudo de Damiani et al. ${ }^{35}$ sobre a importância do alcoolismo crônico e excessivo na incidência de aterosclerose carotídea, também realizado na Liga de Aterosclerose da Santa Casa de São Paulo, mas em período anterior (1991-1994), foi difícil selecionar pacientes etilistas crônicos "puros", já que a maioria deles apresentava outros fatores de risco, especialmente HAS e tabagismo. Nesta pesquisa, os autores observaram que, entre pacientes do sexo masculino e com média de idade de 60 anos, o uso excessivo e continuado de bebidas alcoólicas associado ao tabagismo e à HAS se relaciona com maior prevalência de ateromas na artéria carótida do que entre pacientes que, a despeito de serem tabagistas e apresentarem HAS, não ingerem bebida alcoólica. Por outro lado, estudo sobre a influência do etanol das bebidas alcoólicas na aterosclerose em artérias carótidas extracranianas em pacientes não tabagistas e normotensos sugeriu ação protetora do álcool etílico para aterosclerose carotídea, quando ingerido em moderada quantidade (ente $1 \mathrm{ml}$ e $300 \mathrm{ml} / \mathrm{semana})^{36}$. A aterosclerose pode acometer diferentes artérias do organismo por mecanismos fisiológicos que, embora semeIhantes, podem apresentar variações importantes dependendo da artéria afetada. No caso da aterosclerose, acredita-se que as artérias carótidas sejam as mais susceptíveis, acarretando problemas cerebrais importantes. Entre os fatores de risco para a aterosclerose, a HAS é o que pode afetar mais precoce e intensamente as artérias carótidas ${ }^{30}$.

Achados anormais à TC e à ultra-sonografia de carótidas foram registrados para $40,8 \%$ e $48,2 \%$ dos pacientes, respectivamente. Dentre os resultados tomográficos disponíveis, em 18 casos eram nor- 
mais, o que nos leva a crer que estas tomografias tenham sido realizadas em fase aguda do acidente, momento ainda precoce para a detecção de anormalidades. Os achados da ultra-sonografia do sistema carotídeo evidenciaram que a grande parte dos pacientes $(43,7 \%)$ em cujo prontuário se encontrava este dado $(65,1 \%)$ apresentava grau de estenose $\leq 50,0 \%$. Rappeport et al. ${ }^{37}$ relataram estenose maior do que $50,0 \%$ em $14,0 \%$ dos pacientes com AVC com menos de 71 anos, relatando taxa de $2,0 \%$ de pacientes para os quais se deveria considerar a realização de endarterectomia.

É importante ressaltar que este é um dos diferentes estudos que vêm sendo desenvolvidos na Liga de Aterosclerose para que se possa delinear o perfil diagnóstico e demográfico dos pacientes com diferentes doenças cerebrovasculares lá cadastrados.

Este estudo sobre as freqüências dos fatores de risco modificáveis para AVCi em população idosa permite concluir que: (1) a freqüência de HAS é significativamente alta nesta população $(87,8 \%)$, independentemente do sexo e da faixa etária; (2) as freqüências de tabagismo e etilismo também são altas neste grupo de pacientes, principalmente no sexo masculino, independentemente de a sua história ser pregressa ou atual; (3) cardiopatias (27,0\%), DM $(19,9 \%)$, e dislipidemia $(15,6 \%)$ também constituem fatores de risco modificáveis freqüentes, independentemente do sexo e da faixa etária dos pacientes idosos; (4) as cardiopatias foram mais freqüentes no grupo de idade mais avançada, embora sem diferença estatisticamente significativa; e (5) embora a hiperuricemia seja achado relativamente freqüente no idoso, a sua ocorrência no grupo de pacientes aqui estudado não se mostrou tão alta.

\section{REFERÊNCIAS}

1. Brasil, Ministério da Saúde - Sistema de informações sobre mortalidade. DATASUS - TABNET. Indicadores e dados básicos. Brasil, 1997. Disponível em: www.datasus.gov.br. 1999.

2. Gomes MM. Doenças do cérebro: prioridade de política de saúde pública no Brasil? Rev Bras Neurol 1992;28:11-16.

3. Adams RD, Victor M, Ropper AH. Cerebrovascular diseases. In: Adams RD, Victor M, Ropper AH (ed). Principles of neurology, 6th ed. New York: McGraw Hill, 1997;777-873.

4. Greenberg DA, Aminoff MS, Simon RP. Neurologia clínica, 2.Ed. Porto Alegre: Artes Médicas, 1996;273-306.

5. Gagliardi RJ, Reimão R. Clínica neurológica. São Paulo: Lemos, 1998.

6. Radanovic M. Características do atendimento de pacientes com acidente vascular cerebral em hospital secundário. Arq Neuropsiquiatr 1999;58:99-106.

7. Lessa, I. Epidemiologia das doenças cerebrovasculares no Brasil. Rev Soc Cardiol Estado S Paulo 1999;4:509-518.

8. SBDCV - Sociedade Brasileira de Doenças Cerebrovasculares. Primeiro consenso brasileiro do tratamento da fase aguda do acidente vascular cerebral. Arq Neuropsiquiatr 2001;59:972-980.

9. Aboderin I, Venables G. Stroke management in Europe: Pan European Consensus Meeting on Stroke Management. J Intern Med 1996;240:173-180.
10. AAN - American Academy of Neurology. Quality Standards Subcommitee of the American Academy of Neurology. Practice advisory: thrombolytic therapy for acute ischemic stroke. Neurology 1996;47:835-839.

11. Gorelick PB. Stroke risk factor modification. 51th Annual Meeting of American Academy of Neurology. Syllabi on CD-ROM, 1999.

12. EUSI - European Stroke Initiative. Recommendations for stroke management. Cerebrovasc Dis 2000;10:1-34.

13. WHO - World Health Organization. The World Health Report. Geneve: WHO, 1997.

14. Weinberger J, Biscarra V, Weisberg MK, Jacobson JH. Factors contributing to stroke in patients with atherosclerotic disease of great vessels: the role of diabetes. Stroke 1983;14:709-712.

15. Akai T, Takahata T, Sugita K, Sonobe M, Takahashi S. The outcome of cerebrovascular disease in the elderly. No To Shinkei 1997;49:337-341.

16. Cabral NL, Longo A, Moro CHC, Amaral CH, Kiss HC. Epidemiologia dos acidentes vasculares em Joinville, Brasil: estudo institucional. Arq Neuropsiquiatr 1997;55:357-363.

17. Johnston KC, Li JY, Lyde PD. Medical and neurological complications of ischemic stroke experience from de RANTTAS trial: TANTTAS investigators. Stroke 1998;29:447-453.

18. Botrel TEA, Costa RD, Costa MD, Costa AMD. Doenças cardiovasculares causas e prevenção. Rev Bras Clin Ter 2000;26:87-90.

19. Lee TH, Hsu WC, Chen CJ, Chen ST. Etiologic study of young ischemic stroke in Taiwan. Stroke 2002;33:1950-1955.

20. Sacco RL, Benjamin EJ, Broderick JP. Risk factors. Stroke 1997;28:1507-1517.

21. Weir CJ, Murray GD, Adams FG, Muir KW, Grosset DG, Less KR. Poor accuracy of stroke scoring ystems for differential clinical diagnosis of intracranial haemorrhage and infarction. Lancet 1994;334:999-1002.

22. Massaro AR. Proposta de uma nova escala de avaliação clínica para o diagnóstico diferencial entre acidente vascular cerebral isquêmico e hemorrágico. Tese, Universidade de São Paulo São Paulo, 1998.

23. Fayad P. Identifying and managing stroke risk factors. 53th Annual Meeting of the American Academy of Neurology. Syllabi on CD-ROM, 2001

24. Abel GA, Sacco RL, Lin I-F. Race-ethnic variability in etiologic fraction for stroke risk factors: the Northern Manhattan Stroke Study. 23 ${ }^{\text {rd }}$ International Joint Conference on Stroke and Cerebral Circulation. Orlando, Fl, 1998. (Program and Abstracts).

25. Zétola VHF, Nóvak EM, Camargo CHF, et al. Acidente vascular cerebral em pacientes jovens: análise de 164 casos. Arq Neuropsiquiatr 2001;59:740-745.

26. SHEP Cooperative Research Group. Prevention of stroke by antihypertensive drug treatment in older persons with isolated systolic hypertension: final results of the Systolic Hypertension in the Elderly Program (SHEP). JAMA 1991;265:3255-3264.

27. Mano R. Conceito atual de hipertensão arterial sistêmica. Disponível em http: / / www.manuaisdecardiologia.med.br/has/Pag17.htm. Acessado em 11 de março de 2003.

28. Moriguti JC, Paiva CE, Marchini JS, Furtado DA Jr, Matos FD, Ferrioll E. Systolic Hypertension in the Elderly Program e outros estudos clínicos em idosos. Rev Bras Hipertens 2001;8:206-211.

29. SBD - Sociedade Brasileira de Diabetes. Diagnóstico e classificação do diabetes mellitus e tratamento do diabetes mellitus tipo 2: recomendações da Sociedade Brasileira de Diabetes, Versão final. Disponível em http. / / www.diabetes.org.br. Acessado em 13 de novembro de 2002.

30. Wolf PA, Abbott RD, Kannel WB. Atrial fibrillation as an independent risk factor for stroke: the Framingham Study. Stroke 1991;22:983-988.

31. Gagliardi RJ, Sanches M, Rasslan Z, Guedes MLS. Comparação das frações HDL e LDL colesterol como fatores de risco para a aterosclerose carotídea. Arq Neuropsiquiatr 1995;53:730-736.

32. Brown MS, Goldstein JL. A receptor-mediated pathway for cholesterol homeostasis. Science 1986;232:34-37.

33. Yatsu FM, Fisher M. Atherosclerosis: current concepts on pathogenesis and interventional therapies. Ann Neurol 1989;26:3-12.

34. Tinsley JA, Finlayson RE, Morse RM. Developments in the treatment of alcoholism. Mayo Clin Proc 1998;73:857-863.

35. Damiani IT, Neme RM, Gagliardi RJ. Importância do alcoolismo crônico e excessivo na incidência de aterosclerose carotídea: pesquisa clínica e epidemiológica. Rev Bras Neurol 1996;32:65-68.

36. Damiani IT. A influência do etanol das bebidas alcoólicas na aterosclerose em artérias carótidas extracranianas. Dissertação, Universidade de São Paulo, São Paulo, 1999

37. Rappeport Y, Simonsen L, Christiansen H, Boysen G. Prevalence of significant carotid artery stenosis in patients with transient ischaemic attack. Med Sci Monit 2002:8:CR317-CR320. 